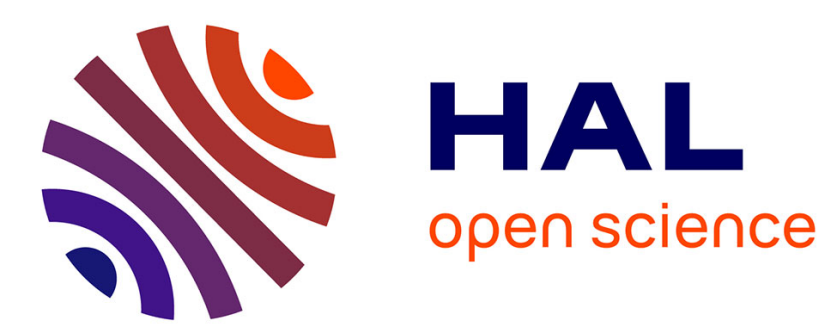

\title{
Applications of the finite volumes method for complex flows: From the theory to the practice \\ Philippe Helluy, Frederic Golay
}

\section{To cite this version:}

Philippe Helluy, Frederic Golay. Applications of the finite volumes method for complex flows: From the theory to the practice. Flow, Turbulence and Combustion, 2006, 76 (4), pp.315-329. hal-00139619

\section{HAL Id: hal-00139619 \\ https://hal.science/hal-00139619}

Submitted on 3 Apr 2007

HAL is a multi-disciplinary open access archive for the deposit and dissemination of scientific research documents, whether they are published or not. The documents may come from teaching and research institutions in France or abroad, or from public or private research centers.
L'archive ouverte pluridisciplinaire HAL, est destinée au dépôt et à la diffusion de documents scientifiques de niveau recherche, publiés ou non, émanant des établissements d'enseignement et de recherche français ou étrangers, des laboratoires publics ou privés. 


\title{
Applications of the finite volumes method for complex flows: from the theory to the practice
}

\author{
Philippe HELLUY (helluy@univ-tln.fr) and Frédéric GOLAY \\ (frederic.golay@univ-tln.fr) \\ Laboratoire ANAM/MNC, ISITV, BP 56 \\ 83162 La Valette CEDEX \\ november 2005
}

Abstract. In this short paper, we recall some well-known results on hyperbolic systems of conservation laws. We introduce the Godunov finite volume scheme for their approximations. We then present two recent applications to multiphase flows: the computation of a wave breaking and the construction of entropic schemes for phase transition.

Keywords: hyperbolic systems, Godunov scheme, finite volumes, two-phase flows, phase transition.

\section{Hyperbolic systems of conservation laws}

Hyperbolic Systems of Conservations Laws (HSCL) provide useful mathematical models in many domains. It is thus important to understand theirs properties and construct efficient numerical approximations.

\subsection{Characteristic Curves}

We consider the simplest HSCL that is the transport equation

$$
w_{t}+u \cdot w_{x}=0,
$$

the unknown is a function $w(x, t), x$ is the space variable and $t$ the time variable. The velocity $u$ is a constant.

A characteristic curve, is a curve in the $(x, t)$ plane along which a solution to (1) is constant. If that curve is parameterized by time $(x(t), t)$, we must have

$$
\begin{aligned}
& \frac{d}{d t} w(x(t), t)=0, \\
& w_{t}+x^{\prime}(t) w_{x}=0 .
\end{aligned}
$$

It is thus natural to take $x^{\prime}(t)=u$. The general equation of characteristics is then $x-u t=C s t$. We deduce that the general solution of (1) is an arbitrary function of $x-u t$.

A more complicated example of HSCL is the Burgers equations

$$
w_{t}+\left(w^{2} / 2\right)_{x}=0 .
$$

(C) 2006 Kluwer Academic Publishers. Printed in the Netherlands. 
A regular solution $w(x, t)$ of that equation also satisfies

$$
w_{t}+w w_{x}=0
$$

The characteristic equation reads here

$$
x^{\prime}(t)=w(x(t), t)=C s t .
$$

A general characteristic admits the parameterization

$$
x(t)=x_{0}+w\left(x_{0}, 0\right) t .
$$

If we consider now an initial condition of the type

$$
w(x, 0)=\left\{\begin{array}{clc}
1 & \text { if } & x<0 \\
1-x & \text { if } & 0 \leqslant x \leqslant 1 \\
0 & \text { if } & x>1
\end{array}\right.
$$

we observe that two characteristics coming respectively from $x_{0}<0$ and $x_{0}>1$ transport the two different values 1 and 0 and necessarily intersect. The notion of classical solution is thus not sufficient and has to be extended.

\subsection{Shock WAVES, ENTROPy CONDitions}

Using the distribution theory [18], it is possible to define discontinuous solutions to (3). The curve along which the solution is discontinuous is called a shock ${ }^{1}$. Let us consider a parametrization of the shock by time $(x(t), t)$. Defining the shock velocity by $s=x^{\prime}(t)$, the normal vector to the shock in the $(x, t)$ plane, oriented from the left $(L)$ to the right $(R)$, is $n=\left(n_{x}, n_{t}\right)=(1,-s)$. We denote by $w_{L}$ the values of $w$ on the left of the shock and by $w_{R}$ the values of $w$ on the right of the shock. We also denote by brackets the jump of a quantity in the shock

$$
[w]=w_{R}-w_{L} .
$$

A weak solution of (3) is then a function $w(x, t)$ that satisfies the Burgers equation in the usual sense where it is regular. In a discontinuity, the solution has to satisfy the Rankine-Hugoniot jump condition

$$
\begin{aligned}
& n_{t}[w]+n_{x}\left[w^{2} / 2\right]=0, \quad \text { equivalent to } \\
& s=\frac{w_{L}+w_{R}}{2} .
\end{aligned}
$$

${ }^{1}$ The shock wave terminology is employed for non linear conservation laws and the Burgers equation (3) is indeed non linear. For the linear convection equation (1) the discontinuous solutions are rather called contact discontinuity waves 
On the other hand, when the solution can be computed by the characteristic method, it is useless to introduce discontinuous solution. A shock wave has to emerge from an intersection of characteristics. In other words, an admissible shock wave has to move slower than the characteristics coming from the left and faster than the characteristics coming from the right. This leads to the Lax admissibility condition $[14]$

$$
w_{L}>s>w_{R} .
$$

The Lax characteristic criterion is a purely geometric criterion. It is often useful to use a criterion based on conservation laws. When the solution is regular, we can deduce from the Burgers equation supplementary conservation laws of the form

$$
U(w)_{t}+F(w)_{x}=0 .
$$

If $w \rightarrow U(w)$ is convex, $U$ is then called a Lax entropy for the Burger equation. The function $w \rightarrow F(w)$ is called the entropy flux. In a shock, the Lax entropy condition requires that

$$
n_{t}[U(w)]+n_{x}[F(w)] \leq 0 .
$$

It is easy to verify that the Lax entropy condition (12) is equivalent to the Lax characteristic condition (10) in the case of the Burgers equation.

For a more detailed presentation, we refer to the original paper of Lax [14], but also to the books of Toro [20] and Godlewski and Raviart $[6]$.

\subsection{Riemann PROBlem}

The Riemann problem consists in finding a weak solution to (3) when the initial condition is made of two constant states. It reads

$$
\begin{aligned}
& w_{t}+\left(w^{2} / 2\right)_{x}=0, \\
& w(x, 0)=\left\{\begin{array}{l}
w_{L} \text { if } x<0, \\
w_{R} \text { if } x>0 .
\end{array}\right.
\end{aligned}
$$

The entropy solution can be computed explicitly. Two cases are possible

- If the initial condition satisfies $w_{L}<w_{R}$ then the solution is a rarefaction wave defined by

$$
w(x, t)=\left\{\begin{array}{ccc}
w_{L} & \text { if } & x / t<w_{L} \\
x / t & \text { if } & w_{L}<x / t<w_{R} \\
w_{R} & \text { if } & w_{R}<x / t
\end{array}\right.
$$


- On the other hand, if $w_{L}>w_{R}$, then the solution is an admissible shock wave

$$
w(x, t)= \begin{cases}w_{L} & \text { if } x / t<s=\frac{w_{L}+w_{R}}{2} \\ w_{R} & \text { if } x / t>s\end{cases}
$$

We observe that the solution of the Riemann problem is self-similar, it is thus noted

$$
w(x, t)=R\left(x / t, w_{L}, w_{R}\right) .
$$

\subsection{Generalizations}

All the previous notions (characteristics, shock waves, Lax conditions, Riemann problem, etc.) can be extended to a general system of conservation laws, $w \in R^{m}, m>1$,

$$
w_{t}+f(w)_{x}=0,
$$

under a condition of hyperbolicity: the jacobian matrix $f^{\prime}(w)$ of the flux vector $f(w)$ is diagonalizable with real eigenvalues for all vectors $w$. The solution of the general Riemann problem is then made of $m+1$ constant states separated by shock, contact discontinuity or rarefaction waves.

The previous notions can also be extended to multidimensional conservation laws. In two space dimensions $(x, y)$ for example, the unknown $w(x, y, t) \in R^{m}$ satisfies

$$
w_{t}+f(w)_{x}+g(w)_{y}=0 .
$$

The flux depends on the direction $\nu=\left(\nu^{x}, \nu^{y}\right)$

$$
F(w, \nu)=f(w) \nu^{x}+g(w) \nu^{y} .
$$

The system is hyperbolic here if $D F / D w$ is diagonalizable with real eigenvalues for all vectors $w$ and all directions $\nu$. The solution of the Riemann problem becomes much more complicated.

\subsection{Godunov scheme}

The Godunov scheme is a numerical method to compute the solutions of (3). It can be generalized, with some adaptations, to (17) and (18). Let $\tau$ be a time step and $h$ a space step. Let $t_{n}=n \tau$ and $x_{i}=i h$. The cells (or finite volumes) centered on the points $x_{i}$ are defined by

$$
\left.C_{i}=\right] x_{i-1 / 2}, x_{i+1 / 2}[.
$$


We look for an approximation $w_{i}^{n}$ of $w\left(x_{i}, t_{n}\right)$ in the cell $C_{i}$ at the time $t_{n}$. The approximation is given by the formula

$$
\frac{w_{i}^{n+1}-w_{i}^{n}}{\tau}+\frac{f_{i+1 / 2}^{n}-f_{i-1 / 2}^{n}}{h}=0 .
$$

The numerical flux $f_{i+1 / 2}^{n}$ is obtained after a resolution of a Riemann problem between the cells $C_{i}$ and $C_{i+1}$

$$
f_{i+1 / 2}^{n}=f\left(R\left(0, w_{i}^{n}, w_{i+1}^{n}\right)\right) .
$$

The scheme is stable under a CFL condition $\tau<h / s \max$, where $s \max$ is the maximal wave speed at time $t_{n}$.

The Godunov scheme is very easy to implement. An example in FORTRAN is given at

$$
\text { http://helluy. univ-tln.fr/PHYP/burgers.f }
$$

It is only first order, but many extensions exist: second order, systems, multidimensional systems, etc. When the Riemann problem is difficult to solve, one can use an approximate Riemann solver. A huge literature has been written on this subject: [7], [21], [9], [5], etc.

\section{Application to two-phase flows}

Before studying the hydrodynamics of a two-phase flow that can be described by a HSCL with source terms, we recall some basic facts on thermodynamics.

\subsection{Entropy of A TWO-Phase miXture}

\subsubsection{Thermodynamics of a single fluid}

Consider a single fluid of mass $M \geq 0$, internal energy $E \geq 0$, occupying a volume $V \geq 0$. If the fluid is homogeneous and at rest, its behavior is entirely defined by its entropy function

$$
S:(M, V, E) \rightarrow S(M, V, E) .
$$

In the sequel, we note $W=(M, V, E)$. The vector $W$ belongs to a closed convex cone of $R^{3}$,

$$
C=\{(M, V, E), M \geq 0, V \geq 0, E \geq 0\} .
$$

According to thermodynamics the entropy function must satisfy

- The entropy $S$ is positively homogeneous of degree 1 (in short: "S is PH1")

$$
\forall \lambda>0, \quad S(\lambda W)=\lambda S(W) .
$$


- The entropy $S(W)$ is concave with respect to $W$.

The chosen axiomatic is justified in [13], [3], [4].

The inverse of the temperature is defined by

$$
\theta=\frac{1}{T}=\frac{\partial S}{\partial E},
$$

the pressure is

$$
p=T \frac{\partial S}{\partial V},
$$

and the chemical potential (or the Gibbs specific energy) is

$$
\mu=-T \frac{\partial S}{\partial M} .
$$

In this way, we recover the classical relation

$$
T d S=d E+p d V-\mu d M .
$$

Euler's relation for PH1 functions lead to $S(W)=\nabla S(W) \cdot W$, and this is nothing else than the Gibbs relation

$$
\mu M=E+p V-T S .
$$

The quantity $G=\mu M$ is called the Gibbs free energy. Usually, the PH1 functions of $W$ are said extensive. The PH0 functions are said intensive. The gradient of a $\mathrm{PH} 1$ function being $\mathrm{PH} 0$, the temperature, the pressure and the chemical potential are necessarily intensive. It is also usual to define the specific entropy $s$ by

$$
M s=S(M, V, E) .
$$

Because $S$ is PH1, we see that $s$ is $\mathrm{PH} 0$ (intensive) and that

$$
s=S\left(1, \frac{V}{M}, \frac{E}{M}\right),
$$

so, it is natural to consider the specific entropy as a function of the specific volume $\tau=V / M$ and of the specific energy $\varepsilon=E / M$. The density is the inverse of the specific volume, $\rho=1 / \tau$. Setting $M=1$ in the previous formula, we see that we also have

$$
T d s=d \varepsilon+p d \tau .
$$

Gibbs relation (29) can also be written

$$
\mu=\varepsilon+p \tau-T s .
$$




\subsubsection{Mixtures}

We consider now two phases, for example a gas (1) and a liquid (2). Each phase is characterized by its own entropy function $S_{i}, i=1,2$.

According to thermodynamics, the mixture entropy $\Sigma$ is the sum of the two entropies. Then, out of equilibrium, it depends on $W_{1}=$ $\left(M_{1}, V_{1}, E_{1}\right)$ and $W_{2}=\left(M_{2}, V_{2}, E_{2}\right)$ in the cone $C$

$$
\Sigma\left(W_{1}, W_{2}\right)=S_{1}\left(W_{1}\right)+S_{2}\left(W_{2}\right) .
$$

Let us now fix the mass, the volume and the energy of the mixture $W=(M, V, E)$. The conservation of mass, volume and energy imply $W=W_{1}+W_{2}$. The entropy is maximal at equilibrium

$$
S(W)=\max _{0 \leqslant W_{1} \leqslant W} S_{1}\left(W_{1}\right)+S_{2}\left(W-W_{1}\right) .
$$

The operation $\left(S_{1}, S_{2}\right) \rightarrow S$ defined in (35) is classical in convex analysis, it is called the sup-convolution. It is deeply linked to the Legendre transformation [11].

At equilibrium of the two phases, using (25), (26), (27), we recover that $T_{1}=T_{2}, p_{1}=p_{2}$ and $\mu_{1}=\mu_{2}$.

It is more practical to work with intensive variables. We define the volume fraction $\alpha=V_{1} / V$, the mass fraction $\varphi=M_{1} / M$ and the energy fraction $z=E_{1} / E$. The fraction vector is defined by

$$
Y=(\alpha, \varphi, z) .
$$

The specific entropy out of equilibrium is given by (31), or

$$
s(\tau, \varepsilon, Y)=\varphi s_{1}\left(\frac{\alpha}{\varphi} \tau, \frac{z}{\varphi} \varepsilon\right)+(1-\varphi) s_{2}\left(\frac{1-\alpha}{1-\varphi} \tau, \frac{1-z}{1-\varphi} \varepsilon\right)
$$

It permits us to define a pressure and a temperature out of equilibrium

$$
\begin{aligned}
& \frac{1}{T}=\frac{\partial}{\partial \varepsilon} s(\tau, \varepsilon, Y), \\
& \frac{p}{T}=\frac{\delta}{\partial \tau} s(\tau, \varepsilon, Y) .
\end{aligned}
$$

The equilibrium specific entropy is obtained after a maximization of the out-of-equilibrium specific entropy with respect to the fractions

$$
\begin{aligned}
& s\left(\tau, \varepsilon, Y_{e q}(\tau, \varepsilon)\right)=\max _{0 \leqslant Y \leqslant 1} s(\tau, \varepsilon, Y), \\
& s(\tau, \varepsilon)=s\left(\tau, \varepsilon, Y_{e q}(\tau, \varepsilon)\right) .
\end{aligned}
$$




\subsection{MODEL}

We propose now the following general model for a $2 \mathrm{D}$ two-phase flow

$$
\begin{aligned}
& w_{t}+f(w)_{x}+g(w)_{y}=\sigma(w), \\
& w=\left(\rho, \rho u, \rho v, \rho \varepsilon+\rho\left(u^{2}+v^{2}\right) / 2, \rho Y\right), \\
& f(w)=\left(\rho u, \rho u^{2}+p, \rho u v,\left(\rho \varepsilon+\rho\left(u^{2}+v^{2}\right) / 2+p\right) u, \rho Y u\right), \\
& g(w)=\left(\rho v, \rho u v, \rho v^{2}+p,\left(\rho \varepsilon+\rho\left(u^{2}+v^{2}\right) / 2+p\right) v, \rho Y v\right), \\
& \sigma(w)=\left(0,0,-\rho g_{0},-\rho g_{0} v,-\lambda\left(Y-Y_{e q}\right)\right) .
\end{aligned}
$$

In this model, the unknowns are the density $\rho$, the velocity vector $(u, v)$, the energy $\varepsilon$ and the fraction vector $Y$ of the two-phase mixture, all depending on the space variables $(x, y)$ and the time $t$. The gravity $g_{0}=9.81 \mathrm{~m} \cdot \mathrm{s}^{-2}$ is constant. The pressure $p$ is defined by (38).

The relaxation parameter $\lambda$ is a positive matrix. The positivity of $\lambda$ implies that the second principle of thermodynamics is satisfied by the model (see [1])

$$
s_{t}+u s_{x} \geqslant 0 .
$$

The eigenvalues of the jacobian of the flux $\frac{D}{D w}\left(f(w) n^{x}+g(w) n^{y}\right)$ are $u n^{x}+v n^{y}-c, u n^{x}+v n^{y}$ and $u n^{x}+v n^{y}+c$, where $c$ is the sound speed of the mixture. The sound speed depends on the pressure $p=p(\tau, \varepsilon, Y)$

$$
c^{2} / \tau^{2}=p p_{\varepsilon}-p_{\tau} .
$$

The sound speed is also expressed with the specific entropy $s(\tau, \varepsilon, Y)$ by

$$
\rho^{2} c^{2}=-T\left(p^{2} s_{\varepsilon \varepsilon}-2 p s_{\tau \varepsilon}+s_{\tau \tau}\right) .
$$

Thanks to the concavity of $(\tau, \varepsilon) \rightarrow s(\tau, \varepsilon, Y)$ the Hessian of $s$ defines a negative quadratic form. We deduce from (43) that the system (40) is indeed a HSCL if the temperature $T$ in the thermodynamic model is $>0$.

\section{Numerical applications}

\subsection{WAVE BREAKING}

Our first application is devoted to the numerical simulation of wave breaking over a submerged reef. The geometry is sketched on Figure 1.

The initial condition is a stable incompressible solitary wave computed thanks to the method of Tanaka [19]. The programs that compute

the free surface profile and the initial velocity field can be downloaded at 
http://helluy.univ-tln.fr/soliton.htm.

\subsubsection{Model}

In this example, the two-phase mixture is made of air and water. We suppose instantaneous relaxation of pressure and temperature and we suppose that there is no mass transfer between the two phases. The relaxation matrix $\lambda$ in (40) is thus

$$
\lambda=\left[\begin{array}{ccc}
+\infty & 0 & 0 \\
0 & 0 & 0 \\
0 & 0 & +\infty
\end{array}\right]
$$

In practice, we do not compute the volume fraction $\alpha$ and the energy fraction $z$. Only the mass fraction $\varphi$ is convected. The pressure Equation Of State (EOS) is then of the form $p=p(\rho, \varepsilon, \varphi)$. A very simple but realistic choice of EOS is the so-called stiffened gas EOS that reads

$$
\begin{aligned}
& p=(\gamma(\varphi)-1) \rho \varepsilon-\gamma(\varphi) \pi(\varphi), \\
& \frac{1}{\gamma(\varphi)-1}=\varphi \frac{1}{\gamma_{2}-1}+(\varphi-1) \frac{1}{\gamma_{1}-1}, \\
& \frac{\gamma(\varphi) \pi(\varphi)}{\gamma(\varphi)-1}=\varphi \frac{\gamma_{2} \pi_{2}}{\gamma_{2}-1}+(\varphi-1) \frac{\gamma_{1} \pi_{1}}{\gamma_{1}-1} .
\end{aligned}
$$

This EOS is very similar to the perfect gas EOS. It has been used by several authors for multi-fluid flows computations [17], [2], etc.

The sound speed for this EOS is given by

$$
c=\sqrt{\frac{\gamma(p+\pi)}{\rho}} .
$$

It is usually admitted in physics that a flow is incompressible if the Mach number $M=\frac{\sqrt{u^{2}+v^{2}}}{c}$ is lower than $1 / 10$. Here, the real (physical) Mach number is much smaller, of the order of $1 / 400 \sim 1 / 1600$. The natural CFL condition for an incompressible flow would lead to the ideal time step

$$
\tau_{i}=\frac{h}{\sqrt{u^{2}+v^{2}}} .
$$

Because we use a compressible solver our time step will rather be

$$
\tau_{c}=\frac{h}{\sqrt{u^{2}+v^{2}}+c} .
$$

We observe that

$$
\tau_{c}=\tau_{i} \frac{M}{1+M} .
$$

It becomes more and more constraining when $M \rightarrow 0$. 
Furthermore, it is known that numerical imprecisions also arise due to the low Mach number of the flow (see for example [8]). For those two reasons we have been led to choose an artificial pressure law where the sound speed is approximately fixed to $20 \mathrm{~m} \cdot \mathrm{s}^{-1}$.

We choose $\gamma_{1}=\gamma_{2}=1.1$ for the air (1) and water (2). Other choices are possible but we observed a faster convergence of the Newton method in the Riemann solver when $\gamma_{1}=\gamma_{2}$. If the sound speed is fixed to $20 \mathrm{~m} . \mathrm{s}^{-1}$ for a pressure of $p=10^{5} \mathrm{~Pa}$, we find the other pressure law coefficients

$$
\begin{aligned}
& \pi_{1}=-0.99636 \times 10^{5} \mathrm{~Pa}, \\
& \pi_{2}=2.63636 \times 10^{5} \mathrm{~Pa} .
\end{aligned}
$$

\subsubsection{Approximation and results}

The numerical method is a simple second order explicit MUSCL finite volumes method applied to the system (40) and (45). We used an exact Riemann solver because we experimented instabilities with several approximate solvers. It is known that the transport equation on $\varphi$ in (40) has to be approximated with care in order to avoid pressure oscillations. Indeed, the transport equation in (40) and the EOS (45) can be written under many different forms on the continuous side. On the discrete side, these forms are not equivalent, and the one that is presented in (40), (45) plays a special role. We used the trick of Abgrall and Saurel described in [17] and [2] in order to get a numerical scheme that preserves the constant velocity and pressure states.

It is then possible to compute the wave evolution. The numerical profiles at times $t=1.2 \mathrm{~s}, t=1.4 \mathrm{~s}, t=1.6 \mathrm{~s}$ and $t=1.8 \mathrm{~s}$ are given on Figures 2, 3, 4 and 5. A more detailed validation is given in [10].

\subsection{Phase transition}

In this section, we give some comments on the computation of phase transition. We suppose that it is instantaneous, in such a way that the matrix $\lambda$ is now

$$
\lambda=\left[\begin{array}{ccc}
+\infty & 0 & 0 \\
0 & +\infty & 0 \\
0 & 0 & +\infty
\end{array}\right] .
$$

For simplicity, we consider a $1 \mathrm{D}$ version of the model $(40)\left(\frac{\partial}{\partial y}=0\right.$ and $v=0$ ), without gravity. Because of the instantaneous relaxation, we indeed study the limit when $\lambda \rightarrow \infty$ of the system (40):

$$
\begin{aligned}
& w_{t}+f(w)_{x}=0, \\
& w=\left(\rho, \rho u, \rho\left(\varepsilon+u^{2} / 2\right)\right), \\
& f(w)=\left(\rho u, \rho u^{2}+p,\left(\rho\left(\varepsilon+u^{2} / 2\right)+p\right) u\right), \\
& p=p\left(\rho, \varepsilon, Y_{e q}(\rho, \varepsilon)\right) .
\end{aligned}
$$




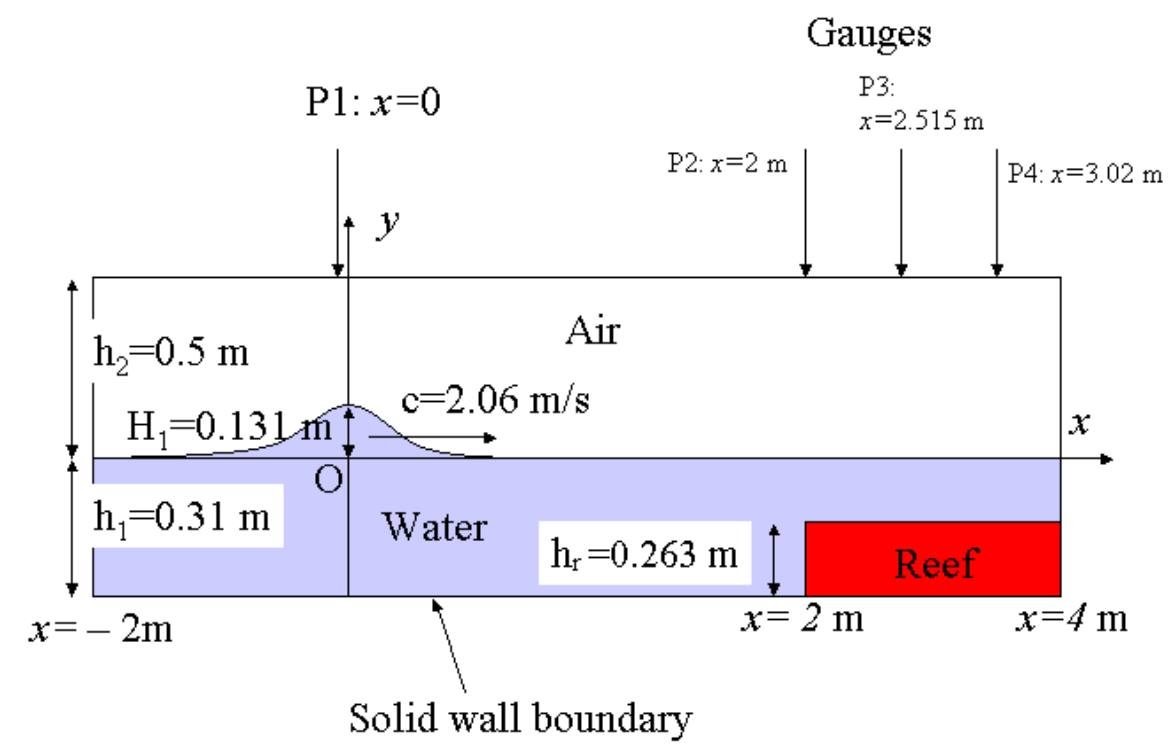

Figure 1. Boundary and initial conditions.

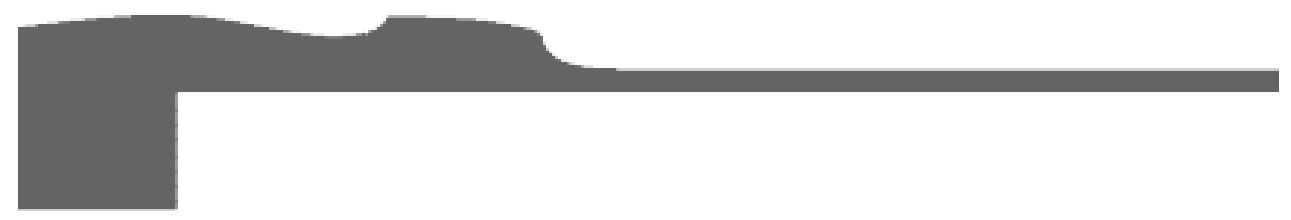

Figure 2. Free surface profile at $t=1.2 \mathrm{~s}$.

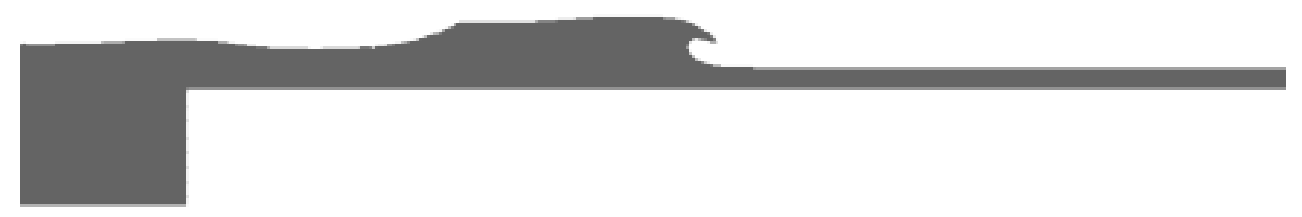

Figure 3. Free surface profiles at $t=1.4 \mathrm{~s}$. 
It appears that the Euler system (52) with the limit pressure law generally possesses several entropy solutions [16]. It is physically reasonable to select the solution that corresponds to the maximal entropy production, also called the Liu's solution [15]. But some classical scheme can converge to wrong entropy solutions [12].

It is interesting to keep the original system (40) in order to design efficient schemes. We have proposed in [1] a simple relaxation scheme. Each time-step is made up of two stages. In the first stage, we solve the system (40) without the source term $\sigma$

$$
\begin{aligned}
& \frac{w_{i}^{n+1 / 2}-w_{i}^{n}}{\Delta t}+\frac{f_{i+1 / 2}^{n}-f_{i-1 / 2}^{n}}{\Delta x}=0, \\
& f_{i+1 / 2}^{n}=f\left(R\left(0, w_{i}^{n}, w_{i+1}^{n}\right)\right) .
\end{aligned}
$$

In order to construct the exact Riemann solver $R$, we consider the system (40) in $1 \mathrm{D}\left(\frac{\partial}{\partial y}=0\right.$ and $\left.v=0\right)$ with the source term $\sigma=0$. See [2] for details.

In the second stage, the density, velocity and energy are kept

$$
\rho_{i}^{n+1}=\rho_{i}^{n+1 / 2}, u_{i}^{n+1}=u_{i}^{n+1 / 2}, \varepsilon_{i}^{n+1}=\varepsilon_{i}^{n+1 / 2},
$$

and the entropy $s$ is optimized with respect to the fractions $Y$ in order to compute $Y_{i}^{n+1}$.

$$
s\left(\rho_{i}^{n+1}, \varepsilon_{i}^{n+1}, Y_{i}^{n+1}\right)=\max _{0 \leqslant Y \leqslant 1} s\left(\rho_{i}^{n+1}, \varepsilon_{i}^{n+1}, Y\right) .
$$

This scheme gives good results. In Figures 6, 7 and 8, we give the result of an academic Riemann problem test case described in [1]. In this case, the two phases satisfy a perfect gas law. The phase entropies are, for $i=1,2$,

$$
S_{i}=M_{i}\left(\ln \frac{E_{i}}{M_{i}}+\left(\gamma_{i}-1\right) \ln \frac{V_{i}}{M_{i}}\right) .
$$

The constants $\gamma_{i}$ are $>1$, for $i=1,2$. The equilibrium pressure law in (52) can be computed. When $\gamma_{1}>\gamma_{2}$, it is given by

$$
\begin{gathered}
p(\tau, \varepsilon)=\left\{\begin{array}{ccc}
\left(\gamma_{2}-1\right) \varepsilon / \tau & \text { if } & \tau \leqslant \tau_{2}, \\
\varepsilon / \kappa & \text { if } \tau_{2} \leqslant \tau \leqslant \tau_{1}, \\
\left(\gamma_{1}-1\right) \varepsilon / \tau & \text { if } & \tau_{1} \leqslant \tau,
\end{array}\right. \\
\tau_{i}=\left(\gamma_{i}-1\right) \kappa . \\
\kappa=\exp \left(1-\frac{\left(\gamma_{1}-1\right) \ln \left(\gamma_{1}-1\right)-\left(\gamma_{2}-1\right) \ln \left(\gamma_{2}-1\right)}{\gamma_{1}-\gamma_{2}}\right) .
\end{gathered}
$$




\section{Conclusion}

In this short review paper, we have given some basic notions on hyperbolic systems of conservations laws. This kind of systems has many applications in physics. It is thus important to design good numerical schemes in order to compute the relevant solutions.

We illustrate the variety of applications by two examples:

- An application to the numerical simulation of wave breaking;

- A simple model for phase transition in order to point out the importance of the notion of entropy and the possibility that standard numerical schemes give wrong solutions.

Of course, there is not enough places here to present the topics in detail. We refer the interested reader to the bibliography...

\section{References}

1. T. Barberon and Helluy. Finite volume simulation of cavitating flows. Computers and Fluids, 34(7):832-858, 2005.

2. T. Barberon, P. Helluy, and S. Rouy. Practical computation of axisymmetrical multifluid flows. International Journal of Finite Volumes, 1(1):1-34, 2003.

3. H. B. Callen. Thermodynamics and an introduction to thermostatistics, second edition. Wiley and Sons, 1985.

4. J.-P. Croisille. Contribution à l'étude théorique et à l'approximation par éléments finis du système hyperbolique de la dynamique des gaz multidimensionnelle et multiespèces. $\mathrm{PhD}$ thesis, Université Paris VI, 1991.

5. Edwige Godlewski and Pierre-Arnaud Raviart. Hyperbolic systems of conservation laws, volume 3/4 of Mathématiques 8 Applications (Paris) [Mathematics and Applications]. Ellipses, Paris, 1991.

6. Edwige Godlewski and Pierre-Arnaud Raviart. Numerical approximation of hyperbolic systems of conservation laws, volume 118 of Applied Mathematical Sciences. Springer-Verlag, New York, 1996.

7. S. K. Godunov. A difference scheme for numerical computation of discontinuous solutions of equations of fluids mechanics. Math Sbornik, n 47:271-306, 1959.

8. Hervé Guillard and Cécile Viozat. On the behaviour of upwind schemes in the low Mach number limit. Comput. Ef Fluids, 28(1):63-86, 1999.

9. A. Harten, P. D. Lax, and B. Van Leer. On upstream differencing and Godunovtype schemes for hyperbolic conservation laws. SIAM Rev., 25(1):35-61, 1983.

10. P. Helluy, F. Golay, and al. Numerical simulations of wave breaking. M2AN, 39(3):591-607, 2005.

11. Jean-Baptiste Hiriart-Urruty and Claude Lemaréchal. Fundamentals of convex analysis. Grundlehren Text Editions. Springer-Verlag, Berlin, 2001.

12. S. Jaouen. Etude mathématique et numérique de stabilité pour des modèles hydrodynamiques avec transition de phase. PhD thesis, Université Paris VI, November 2001. 
13. L. Landau and Lifchitz E. Physique statistique. Physique théorique. Ellipses, Paris, 1994.

14. P.D. Lax. Hyperbolic systems of conservation laws and the mathematical theory of shock waves. In CBMS Regional Conf. Ser. In Appl. Math. 11, Philadelphia, 1972. SIAM.

15. T. P. Liu. The Riemann problem for general systems of conservation laws. $J$. Diff. Equations., 56:218-234, 1975.

16. R. Menikoff and B. J. Plohr. The Riemann problem for fluid flow of real materials. Rev. Modern Phys., 61(1):75-130, 1989.

17. R. Saurel and R. Abgrall. A simple method for compressible multifluid flows. SIAM J. Sci. Comput., 21(3):1115-1145, 1999.

18. Laurent Schwartz. Méthodes mathématiques pour les sciences physiques. Hermann, Paris, 1983.

19. M. Tanaka. The stability of solitary waves. Phys. Fluids, 29:650-6555, 1986.

20. E. F. Toro. Riemann solvers and numerical methods for fluid dynamics, 2nd edition. Springer, 1999.

21. B. Van Leer. Towards the ultimate conservative difference scheme. a second order sequel to the godunov's method. Journal of Computational Physics, 32:101-136, 1979. 


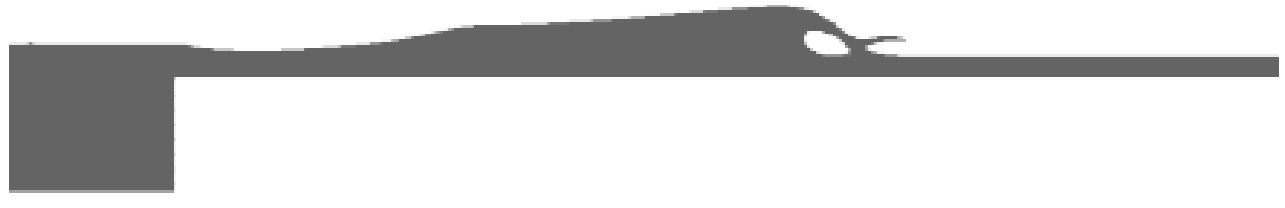

Figure 4. Free surface profiles at $t=1.6 \mathrm{~s}$.

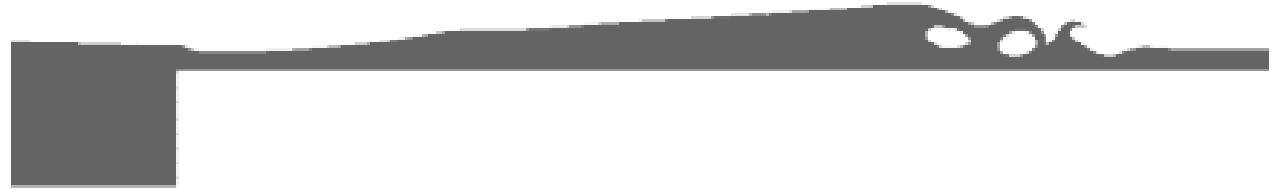

Figure 5. Free surface profiles at $t=1.8 \mathrm{~s}$.

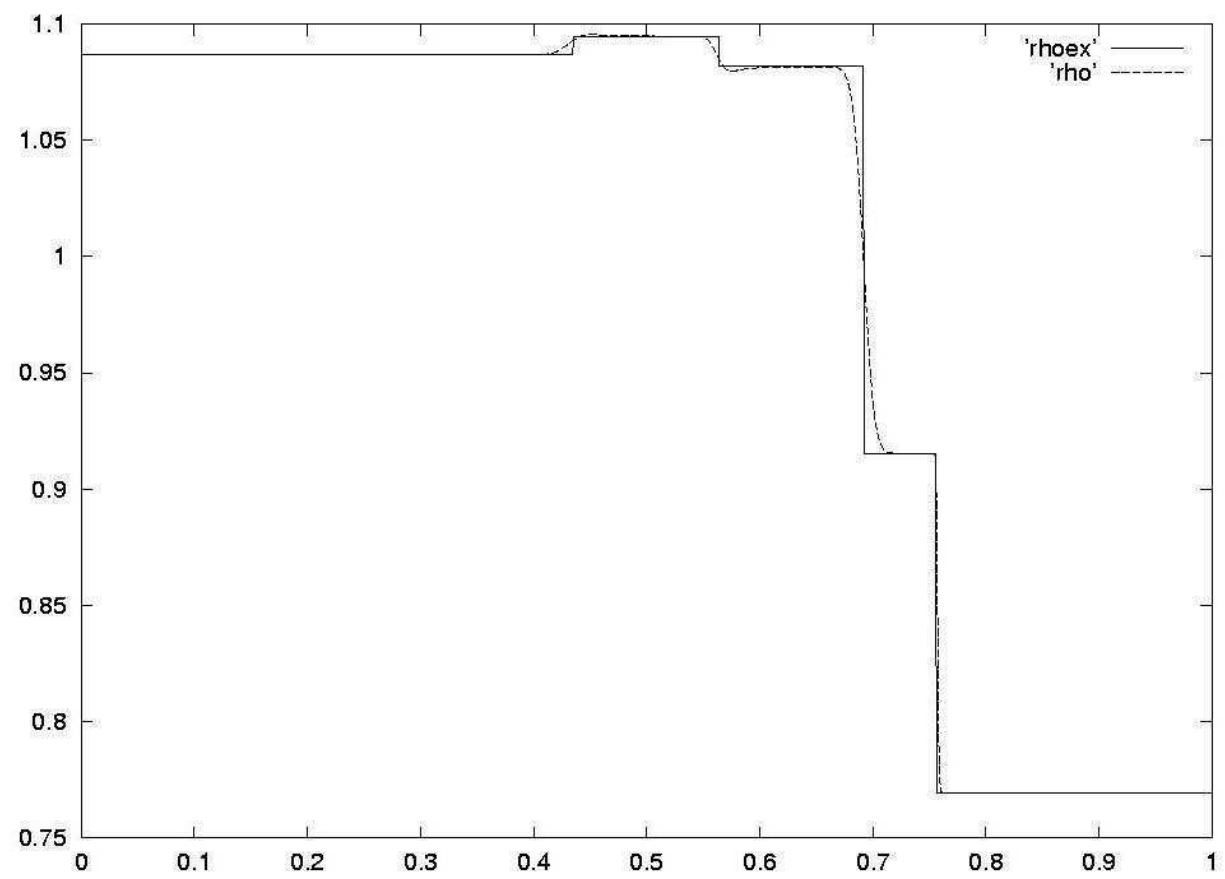

Figure 6. Density. 


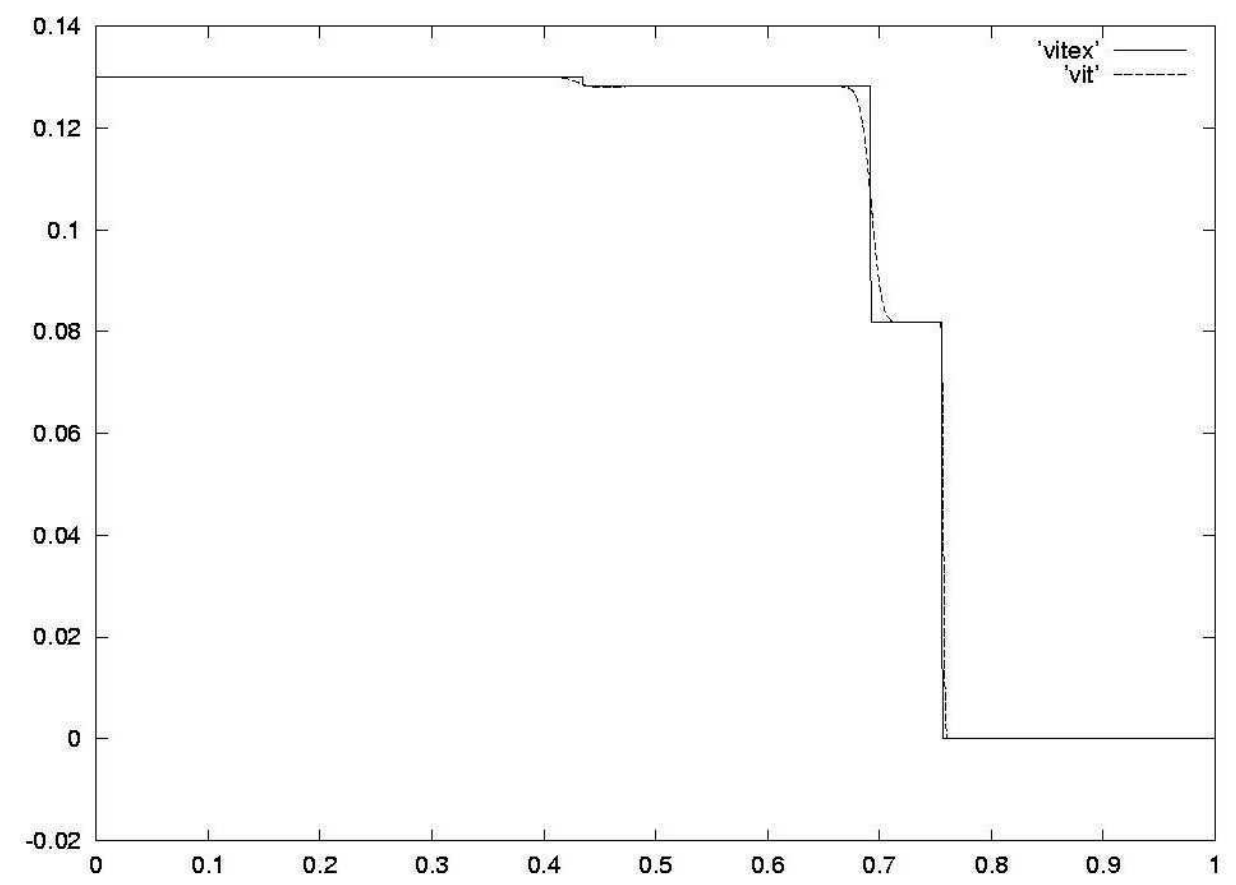

Figure 7. Velocity.

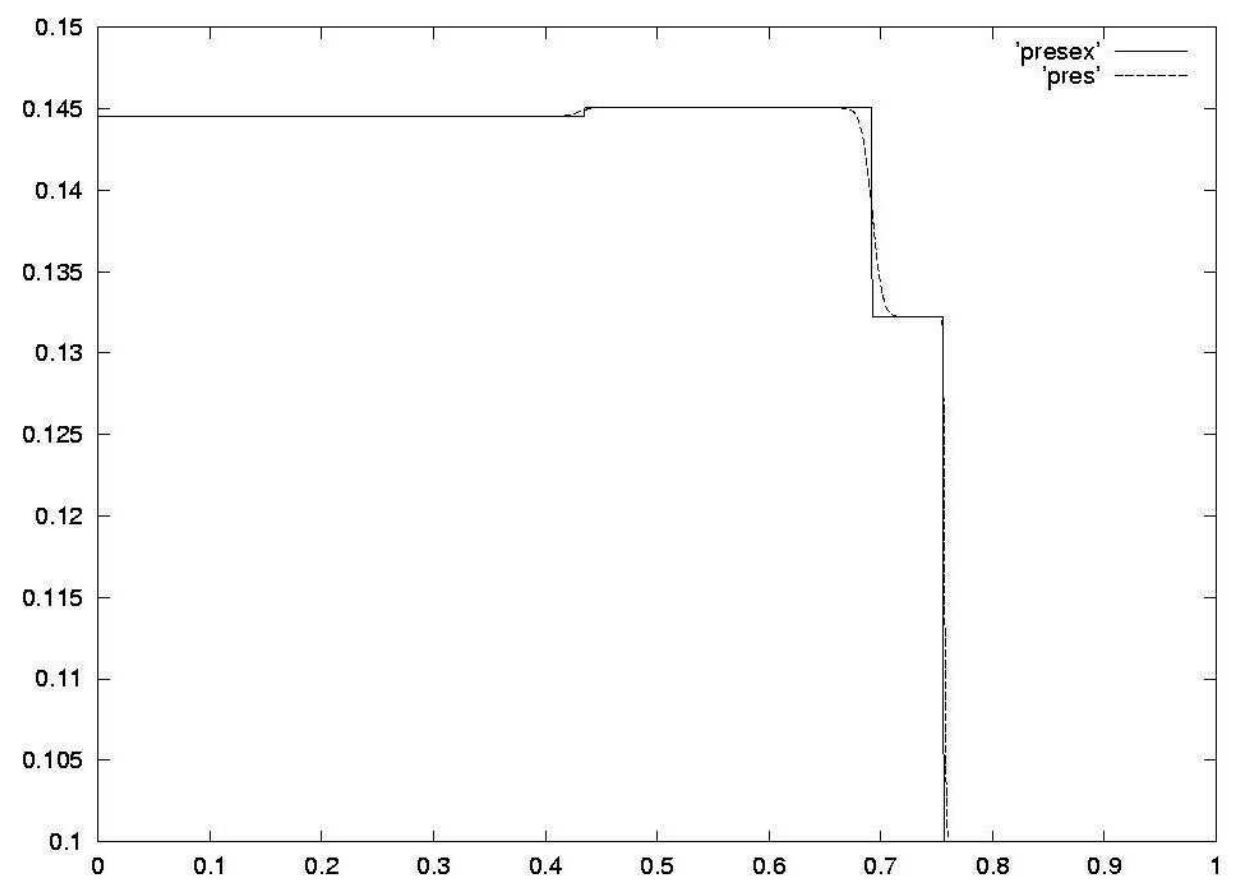

Figure 8. Pressure. 